\title{
EchoGéo
}

$13 \mid 2010$

Afrique, 50 ans d'indépendance : État et territoires

\section{« Le goût amer de la couleur au Cap »}

\section{Chloé Buire}

\section{(2) OpenEdition}

Journals

Édition électronique

URL : https://journals.openedition.org/echogeo/11939

DOI : 10.4000/echogeo.11939

ISSN : 1963-1197

\section{Éditeur}

Pôle de recherche pour l'organisation et la diffusion de l'information géographique (CNRS UMR 8586)

\section{Référence électronique}

Chloé Buire, « "Le goût amer de la couleur au Cap » », EchoGéo [En ligne], 13 | 2010, mis en ligne le 20 septembre 2010, consulté le 03 août 2021. URL : http://journals.openedition.org/echogeo/11939 ; DOI : https://doi.org/10.4000/echogeo.11939

Ce document a été généré automatiquement le 3 août 2021

EchoGéo est mis à disposition selon les termes de la licence Creative Commons Attribution - Pas d'Utilisation Commerciale - Pas de Modification 4.0 International (CC BY-NC-ND) 


\title{
«Le goût amer de la couleur au Cap »
}

\author{
Chloé Buire
}

1 Le Cap fut une escale de ravitaillement hollandaise sur la route des Indes, une tête de pont coloniale britannique en Afrique australe, un cœur politique pour le gouvernement de l'apartheid et est aujourd'hui la vitrine du pays d'Afrique le plus intégré au système monde. Depuis l'installation des premiers Européens en 1652, Le Cap est la "ville-mère" (Mother City) de la nation sud-africaine. Mais cette maternité dont s'enorgueillissent les guides touristiques a ses revers. Aujourd'hui encore, les enfants nés de ce port à la croisée des continents sont en quête œdipienne : l'amour se mêle à la haine et les frères se déchirent pour décider qui est le fils préféré de cette mère équivoque. En 1948, les descendants des Hollandais (afrikaners) ont inventé l'apartheid pour affirmer leur identité sur le mode d'une exclusivité raciale violente et autoritaire. Aujourd'hui, le factionnalisme politique au sein de l'African National Congress (ANC) se teinte d'allégeances ethniques, loin du socialisme panafricain des années de lutte. Dans cet échiquier, Le Cap semble faire bande à part. La métropole de Bonne Espérance hésite : en 2002, l'ANC a dû s'allier à l'ancien parti blanc pour faire éclater la coalition qui avait pris le contrôle de la ville en 2000 sous l'égide de la Democratic Alliance (DA) ${ }^{1}$. En 2007, la municipalité retourne sous la coupe de la DA, domination confirmée à l'échelle provinciale en 2009. Le Cap est donc le vilain petit canard d'un pays autrement dominé par l'ANC. Et ce vote dissident est généralement attribué à la démographie originale de la ville : cas unique en Afrique du Sud, la majorité des habitants est non pas 'africaine' mais 'colorée'2. Le groupe 'coloured' a été officialisé dans la classification raciste de 1950 pour regrouper ceux qui, selon la terminologie officielle d'alors, «ne sont considérés ni comme Blancs ni comme Indigènes $»^{3}$.

2 À travers la question 'colorée' au Cap, cet article propose de saisir des processus identitaires cruciaux pour les enjeux politiques plus larges de l'Afrique du Sud aujourd'hui. Dans les pas de Zimitri Erasmus, il semble en effet être temps de «reconnaître les sentiments et pratiques racistes comme faisant partie de la vie quotidienne et de la construction de tous les individus " afin d'ouvrir la voie à des "positionnements progressistes et transformateurs " ${ }^{4}$. Il s'agira dans un premier temps de comprendre comment au fil des siècles, la société urbaine du Cap a été marquée par la racialisation des relations de classe. Puis, le récit de deux mythes fondateurs, celui 
des forced removal et celui de la hiérarchie des races aidera à comprendre l'identité du groupe 'coloré' comme un positionnement dans la ville, à la fois socialement, spatialement et politiquement. Enfin, le témoignage de Eugene, raconté dans un document vidéo de 7 minutes, mettra en perspective les différents statuts du discours dans la parole scientifique. Prétendre déconstruire un processus identitaire oblige en effet le chercheur à interroger son propre positionnement.

\section{Histoires urbaines : la racialisation des relations de classe au Cap}

Illustration 1- Bo Kaap, dernier quartier de working class dans le centre-ville du Cap ?

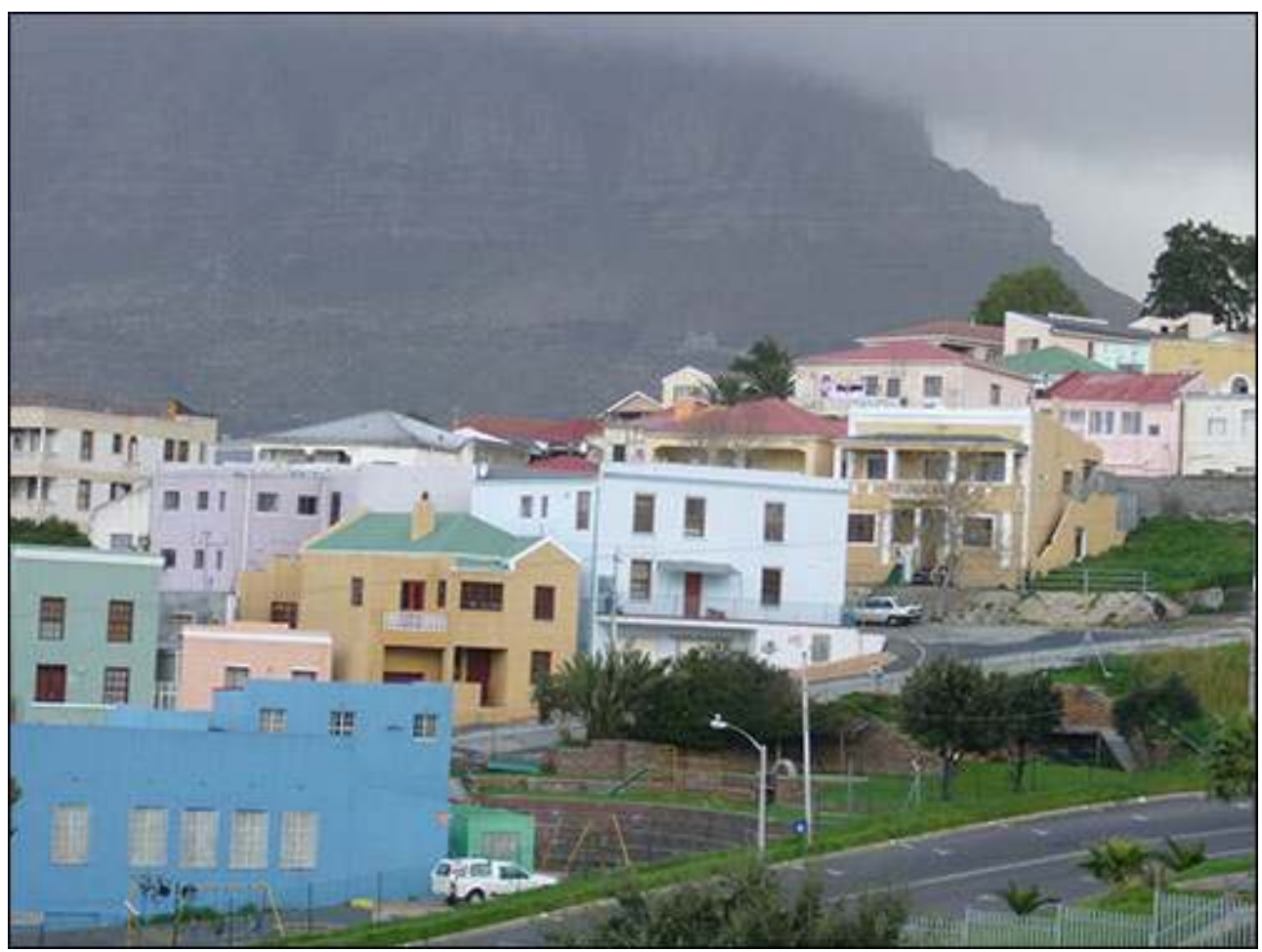

Auteur : Chloé Buire. Bo Kaap, septembre 2008.

3 Le quartier Bo Kaap contraste avec le reste du centre-ville : rues pavées et escarpées grimpant à flanc de colline, petites maisons aux façades colorées anciennement partagées par plusieurs familles. Aujourd'hui le quartier est un modèle de gentrification, les cabanons d'arrière-cour se transforment en chambres d'hôtes, les stoeps, porches autrefois séparés de la rue par un petit muret deviennent des vérandas dûment fermées et sécurisées. Mais Bo Kaap fait partie des attractions touristiques en tant que dernier témoin de la présence des 'classes laborieuses' en ville. Un petit musée y a été installé, dédié "à ceux qui ont construit la ville du Cap, mais n'étaient pas autorisés à profiter des fruits de leur travail». Les photographies narrent la vie du quartier avant 1948: «La discrimination raciale au Cap était une réalité de fait bien avant l'apartheid. Mais l'existence de grands quartiers ouvriers comme Waterkant, BoKaap et District 6, ainsi que de nombreux secteurs plus petits, faisait que les 
habitants noirs ${ }^{5}$ pouvaient quand même avoir une vie culturelle riche dans la ville, malgré les restrictions raciales ».

4 Le discours du musée de Bo Kaap insiste sur une société urbaine mêlant rapports de classe et rapports de race bien avant 1948. Lorsque le Population Registration Act impose des groupes raciaux en 1950, le principe même de différences raciales est intériorisé de longue date, l'Assemblée s'accorde même pour laisser chaque recenseur user de son «bon sens » pour déterminer la race d'un individu, selon son "l'apparence » et "l'acceptation générale " ${ }^{6}$. Bien que l'historiographie du Cap souligne la constitution d'une société urbaine cosmopolite (Worden et al., 1998 et 1999), mise à distance, hiérarchie et peur caractérisent la stratification sociale du Cap depuis le XVII ${ }^{\mathrm{e}}$ siècle.

Dès le recensement de 1731, la trame d'une classification selon les races et les statuts sociaux se fait jour. Il y a les Européens et les autres. Parmi les Européens - venus de Hollande, d'Allemagne, de Russie, de France etc. - l'on distingue les employés de la compagnie et les 'citoyens libres' (free burghers), généralement d'anciens employés ayant choisi de rester au Cap à la fin de leur contrat. Parmi les non-Européens, la classe de 'Noirs libres' a quelque chose d'un creuset culturel. Mais à part quelques 'indigènes' ayant choisi de faire commerce avec les navigateurs, ces 'free Blacks' ont été amenés des possessions hollandaises. Ils se retrouvent 'libres' au Cap après avoir purgé leur peine de prisonniers, ou à la faveur d'une émancipation. L'image d'une ville cosmopolite ne peut faire oublier que la majorité des 'Noirs' sont avant tout des esclaves. Domestiques auprès des 'citoyens' ou des 'noirs libres', main d'œuvre pour la Compagnie, les esclaves d'origines diverses représentent près d'un tiers des habitants du Cap jusqu'à ce que l'abolition progressive de l'esclavage signe leur intégration à la catégorie 'Coloured' qui apparait dans le recensement de $1840^{7}$.

6 L'esclavage est au cœur du développement de la 'Ville-Mère' et les 'Colorés' en sont le produit. Cette réalité souvent évincée par la mémoire nationale plus préoccupée du face à face entre 'Blancs' et 'Noirs' est primordiale dans la difficile affirmation positive des 'Colorés'. Ils sont souvent considérés comme un groupe sans traditions par rapport aux ethnogenèses xhosa, zouloue, afrikaner, voire britannique. D.-C. Martin insiste sur la nécessité d'en finir avec une réduction de l'identité 'colorée' à un métissage qui n'aurait d'autres conséquences que biologiques, l'esclavage est une expérience radicale, une « mort sociale » qui retire aux individus jusqu'à leur nom (Martin, 1998).

7 Après l'abolition (1834), la hiérarchie se reproduit en faveur d'une «élite aisée racialement plus exclusive [que la] population plus pauvre et plus complexe racialement. $»^{8}$. Politiquement, le vote censitaire dénie de facto l'auto-détermination des classes les moins aisées. Économiquement, des spécialisations professionnelles se confirment, avec la communauté de pêcheurs issue des premières familles de 'Noirs libres' par exemple. La différenciation se fait donc spatiale avec la constitution de quartiers populaires où se concentrent les plus précaires. Même l'afrikaans, résultat linguistique de la créolisation du hollandais et signe d'une acculturation séculaire n'est pas unanimement partagé entre les 'Colorés' et les anciens maîtres. L'afrikaans des faubourgs est désormais mâtiné d'anglais, dont la tutelle britannique a imposé l'usage au XIXe siècle (Martin, 1998).

8 Ainsi à la veille de l'apartheid, la classe d'ouvriers et d'artisans à laquelle est dédié le musée de BoKaap regroupe des individus politiquement marginalisés, concentrés dans des quartiers spécifiques et marqués par l'inertie de leur précarité économique. Aux yeux de la bourgeoisie urbaine, ils forment un univers sale et vulgaire, sans lien avec la 
culture européenne mais également distinct des 'Africains'. Pour ces derniers, la municipalité a déjà construit des quartiers périphériques, véritables réservoirs de main d'œuvre réservés aux 'Indigènes' considérés comme inadaptés à la ville'. Mais que faire des citadins qui ne sont pas 'blancs' mais pas 'africains' non plus? Ils n'ont pas de « village » où l'on pourrait les « renvoyer » comme l'impose la création des bantoustans pour les 'Africains'. Ces poches de pauvreté sont des tours de Babel qui défient l'idée même de race. Fidèle à son programme de "développement séparé » (sens littéral du mot 'apartheid'), le Parti national élu en 1948 instaure donc des townships spécifiques aux 'Colorés'. Le Group Areas Act de 1950 impose le déplacement de tous ces «ni noirs ni blancs » à quelques kilomètres de la montagne de la Table contre laquelle s'adossent les suburbs 'blancs'. Les townships remplissent un double objectif aux yeux du gouvernement raciste. D'une part, en transformant le prolétariat urbain en un groupe racial distinct des Blancs, ils sont la réalisation concrète de ce qui sera décrit plus tard comme un «fordisme racial»: l'encadrement de la main d'œuvre ('noire') permet d'augmenter la productivité, soutenue en retour par la consommation croissante d'une classe moyenne montante ('blanche') ${ }^{10}$. En cela l'existence même des townships est une affirmation symbolique: un quartier ouvrier ne peut pas être 'blanc', par définition. D'autre part, ils assurent une zone tampon entre les 'Blancs' et les 'Africains'.

\title{
«Une géographie culturelle hors du commun »
}

\author{
« Auparavant, l'intégration raciale et \\ résidentielle tendait à créer des cultures urbaines \\ par classe sociale (...) Mais la concentration \\ autoritaire de la population africaine dans un \\ quartier a diminué ses possibilités d'interaction \\ avec les autres communautés. Relations sociales, \\ éducation, engagements politiques se feront \\ désormais à l'échelle du township, donnant \\ naissance à une géographie culturelle hors du \\ commun. ( (Houssay-Holzschuch, 1999-a : 73).
}

9 La géographie culturelle « hors du commun » dont parle Myriam Houssay-Holszschuch nourrit aujourd'hui un tourisme d'un genre nouveau : les visiteurs sont encouragés à participer à un township tour, visite guidée d'un ou plusieurs townships, dûment encadrée par un guide, de préférence lui-même 'noir' ou 'coloré'. Visiteurs et organisateurs sont conscients de l'ambiguïté mais veulent croire en une "expérience authentique" : "With Camissa you are not a voyeur or a "tourist", you're our guest. We live here... in the townships. We invite you into our neighbourhood and into our lives.", promet l'agence Camissa Township Tour. La mise en spectacle touristique prouve l'immobilisation des représentations culturelles attachées aux townships et la spatialisation des stéréotypes raciaux (Hammet \& Jayawardane, 2009; Witz, 2008; Buire, 2007). 
Illustration 2 - Géographie intuitive des townships : l'intériorisation des divisions raciales héritées de l'apartheid

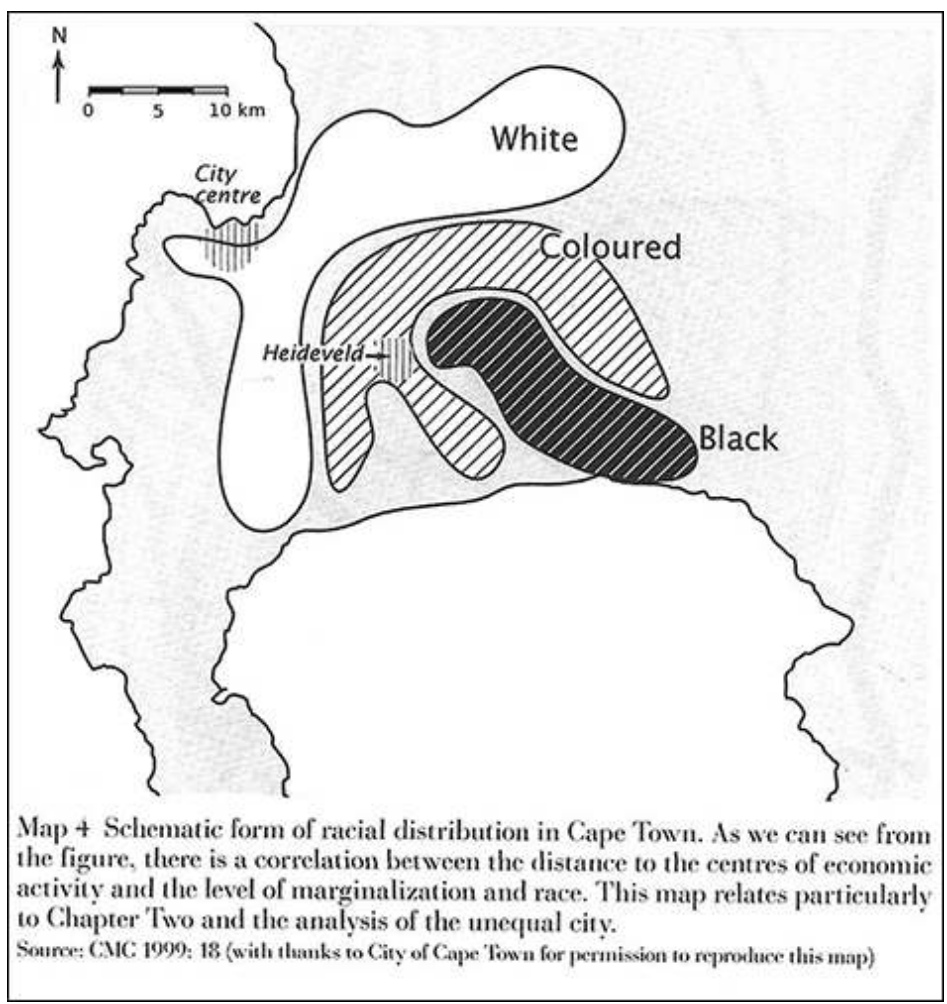

L"échelle, ajoutée par l'auteure, ne doit être utilisée que pour se faire une idée des distances.

Source : Carte tirée de Gangs, Politics \& Dignity in Cape Town, (Jensen, 2008)

Ce schéma caricatural n'est pas une cartographie de la ségrégation mais la carte mentale qui articule les aires intuitives de socialisation et de déplacements selon que l'on se reconnaît de tel ou tel groupe à travers Le Cap. Son utilisation jusque dans une publication scientifique rappelle que l'anthropologue, pas plus que quiconque ne peut faire fi de ces représentations.

Intériorisation des frontières raciales dans la ville, patrimonialisation de l'authenticité des townships, la " géographie culturelle hors du commun » mérite d'être déconstruite plus avant. Comme nous l'avons vu, les discussions sur l'identité 'colorée' partent du constat de l'indétermination raciale de ce groupe " pas assez blanc ni assez noir " (Adhikari, 2005). Or à l'heure de la Nation Arc-en-Ciel, si les hiérarchies sont abolies, les distinctions persistent. De nombreux discours anti-racistes retracent les généalogies diverses de cette "communauté ». Il y a les "Malais du Cap », traditionnellement musulmans même si le terme est convenu pour désigner les descendants d'esclaves en général; il y a aussi ceux qui revendiquent le statut de "peuple premier" en reconstituant une hypothétique ascendance dans les groupes de chasseurscueilleurs San, Nama ou Griqua qui peuplaient la région du Cap à l'arrivée des Européens; il y a encore les métis qui vont puiser dans les textes sur la créolisation pour se réconcilier avec le croisement génétique qui était devenu criminel sous l'apartheid selon «la Loi sur l'Immoralité ». Toutes ces subtilités généalogiques, bien qu'elles démontrent l'ineptie de la classification raciste monolithique de l'apartheid, peinent à ouvrir une réflexion échappant au registre racial et perpétuent l'idée de la colouredness comme le résultat d'un mélange de cultures plus pures ${ }^{11}$. 
Stuart Hall parle d'identités culturelles qui «loin d'être fixées pour l'éternité dans quelque passé essentialisé, (...) sont sujettes au jeu "continu" de l'histoire, de la culture et du pouvoir. Loin d'être fondées sur une simple "redécouverte" du passé, qui attendrait d'être faite, et qui, lorsqu'elle le serait, assurerait pour l'éternité notre sentiment d'être nous-mêmes, les identités sont les noms que nous donnons aux diverses façons d'être situés par les récits du passé et de nous y situer. » (Hall, 2007 : 230). Les identités culturelles sont ainsi considérées comme des processus continus plutôt que comme des produits finis. Mais S. Hall fait un pas de plus en insistant sur la valeur irrémédiablement politique des constructions identitaires issues de "l'expérience coloniale», "situées et assujetties dans les régimes dominants de représentation " (ibid.). Il propose le concept de positionnement pour rendre compte de la dimension stratégique des déterminations identitaires qui «repositionnent en permanence les frontières de la différence par rapport aux différents points de référence » (ibid. : 232). Dans le cas qui l'occupe - les identités culturelles caribéennes, St. Hall identifie trois "présences " par rapport auxquelles se joue le positionnement " caribéen » : la présence africaine, la présence européenne et la présence américaine ${ }^{12}$. C'est dans cet ordre d'idée que je propose de penser l'identité 'colorée' en terme de positionnement face à deux grands récits de référence : les forced removals et le double préjugé racial d'une infériorité par rapport aux 'Blancs' et d'une supériorité par rapport aux 'Africains'.

\title{
Être 'Coloré', un enjeu de positionnement politique
}

\author{
«Les identités culturelles sont les points instables \\ d'identification ou de suture au cœur des discours \\ culturels ou historiques. Non pas une essence, \\ mais un positionnement. » (Hall, $2007: 231$ )
}

13 Le premier grand "discours historique » sur l'identité 'colorée' à Cape Town est celui des forced removals, c'est à dire des expulsions organisées par le gouvernement afin de réaliser son idéal ségrégationniste selon les territoires raciaux définis par le Group Areas Act. Pour reprendre l'expression de N. Murray, D. Shepherd et M. Hall, la victime de forcedremovals est «la figure urbaine emblématique» du Cap ${ }^{13}$. Dans les années 1980 fleurit une littérature critique qui analyse le déracinement des habitants 'colorés' expulsés de la ville centre. John Western documente la transformation de Mowbray, suburb péricentral déclaré quartier 'blanc' (Western, 1981). Au moment de son enquête (1976), un 'Coloré' sur six avait été ainsi déplacé. Don Pinnock consacre une monographie au quartier central de District Six (Pinnock, 1989) rasé dans les années 1980 (plus de 60000 personnes expulsées) et resté vacant jusqu'à aujourd'hui, cicatrice incontournable à deux pas du centre-ville. Les déménagements forcés constituent la trame narrative pour décrire la ville créée par l'apartheid en général, et l'identité des 'colorés' en particulier ${ }^{14}$. J. Western et D. Pinnock décrivent par le menu comment les réseaux sociaux ont été détruits, comment "la communauté "colorée" a été disloquée entre les townships dispersés sur les Cape Flats, hermétiquement démarqués par les no man's land.

«By removing the Coloureds from District Six, the Whites are doing more than

clearing slums or underpinning their exclusive claim to central Cape Town's sacred

space. They are also destroying one of the symbols of whatever Coloured identity may exist, 
a space in parts at least seven generations deep and with associations with the emancipation

of the slaves. » (Western, $1996: 150$, italiques dans l'original)

Les mots de J. Western montrent comment « être 'coloré"» fait moins référence à un contenu qu'à l'enveloppe supposée autour du vide laissé par les déplacements forcés. En ce sens il s'agit bien d'un positionnement qui tente de « suturer » le discours des forced removals. Le musée de District Six en centralisant les paroles des anciens résidents expulsés, des historiens et des militants anti-racistes a lui aussi une valeur performative pour cette affirmation. Non seulement "une identité colorée quelle qu'elle soit » existe (whatever Coloured identity may exist) mais elle est à l'image de ce que fut District Six : une "hybridité » caractérisée par une grande capacité d'adaptation, l'entraide, l'harmonie sociale et le respect de la différence (Soudien, 2001). Encouragés par le musée, d'anciens résidents sont promus « agents d'éducation » (Education Officer). Noor Ebrahim par exemple a publié son témoignage de la communauté détruite (Ebrahim, 1999), emboîtant le pas à des écrivains emblématiques comme Richard Rive ou Alex La Guma. Comme le précisent S. Field, R. Meyer et F. Swanson, le mythe populaire joue un rôle thérapeutique en "procurant aux gens un vocabulaire et des croyances pour comprendre et faire face à une myriade de défis à travers la ville ${ }^{15}$ ». Et le mythe est d'autant plus fort qu'est profond le sentiment d'injustice lié à la vie actuelle dans les townships. P. Gervais-Lambony souligne l'importance de cette nostalgie dans l'édification d'identités spatiales. "Mal du pays", "regret de l'espace d'hier" ou "nostalgie de celui qui regrette de n'avoir jamais connu tel lieu qu'il voit aujourd'hui si différent", la notion de nostalgie conjugue espace et temps et est au cœur de "l'invention de vrais territoires par les hommes" par-delà les incomplétudes du quotidien (Gervais-Lambony, 2003: 139-145). On comprend alors que le récit des déménagements forcés, en idéalisant le passé, raconte aussi l'humiliation d'aujourd'hui inscrite dans l'architecture de contrôle qui marque les paysages. Pour les zones les plus défavorisées des townships 'colorés', l'apartheid a érigé de grands ensembles d'immeubles de trois étages, rangés deux par deux dans le paysage uniformément triste des plaines sableuses balayées par le vent. Ces logements sociaux participent à la reproduction de relations de voisinage marquées par la violence et la suspicion. La quintessence de l'identité 'colorée' au Cap est souvent ramenée aux gangs qui se disputent le contrôle de ces council flats. 
Illustration 3 - Clichés du quotidien à Heideveld, township coloré

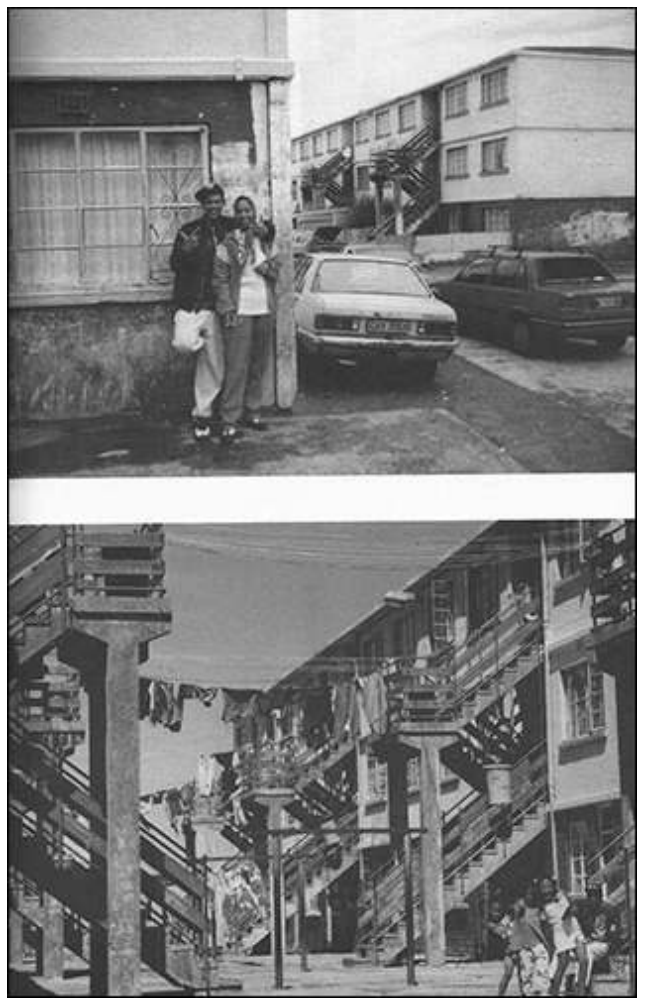

Source : photographies extraites du livre Gangs, Politics \& Dignity in Cape Town (Jensen, 2008 : page non numérotée)

Steffen Jensen introduit son livre consacré aux flats de Heideveld avec une série de photographies prises par les jeunes auprès desquels il a conduit son enquête ethnographique. Bien que non légendées, ces photos donnent un aperçu de l'architecture de type "panoptikon", chaque paire d'immeuble créant une cour centrale où sont braqués les regards du voisinage. Le huis clos participe du rite de passage que constitue l'entrée dans les gangs pour les adolescents de ces quartiers (Pinnock, 1997). On reconnaît sur la première photo l'occupation des « coins » (corners) d'immeubles par des groupes d'adolescents, territorialité on ne peut moins neutre car comme le rappelle S. Jensen "standing on a corner would be reason enough to seriously question the boy's morality (Jensen, $2008: 97$ ).

Décrivant l'indélébile suspicion de délinquance associée aux 'Colorés' du Cap, S. Jensen remonte le fil du deuxième récit de référence, celui d'un positionnement racial. À l'intériorisation de l'infériorité par rapport aux 'Blancs' se mêle la conscience d'un statut privilégié des 'Colorés' par rapport aux 'Noirs'. Un racisme profond entache toutes les relations jusqu'à aujourd'hui. Le sentiment politique le plus répandu dans les townships 'colorés' est celui d'une relégation insultante de la part du nouveau gouvernement qui ne fait rien pour eux. A ce constat, se mêle la honte ambiguë attachée aux statistiques électorales qui rappellent depuis 1994 que les électeurs 'colorés' votent toujours "pour les Blancs" (c'est à dire pour la DA) et sont l'explication de l'exception capetonienne dans la carte électorale du pays.

17 Pour aborder cette question, S. Jensen contourne l'habituel éloge des métissages génétiques associé à l'identité 'colorée' en montrant que c'est précisément l'idée même de « mélange » qui a renforcé les théories raciales. Dès 1937, un rapport sur les 'Colorés' 
du Cap affirmait que "l'impureté » génétique (miscegenation) était source d'une «culture de la pauvreté » et justifiait «scientifiquement» les stéréotypes racistes de son époque (Jensen, 2008). Fort de ce darwinisme, l'État a développé des mesures paternalistes en faveur du groupe 'coloré' : aides sociales et accès privilégié à l'emploi au nom de la Coloured Labour Preference Policy. En instaurant des zones d'emplois réservés aux 'Colorés' à partir de 1955, cette loi visait à la fois à protéger les 'Colorés' de la concurrence des 'Africains', ainsi maintenus hors de la région du Cap et à protéger les 'Blancs' de la concurrence des 'Colorés' puisque seuls les emplois les moins qualifiés étaient concernés. Le principe de 'protection' accordée au groupe 'coloré' montre que le positionnement racial par rapport aux 'Blancs' est indissociable du positionnement par rapport aux 'Africains'. Le complexe identitaire est toujours double « not only not white, but less than white; not only not black, but better than black» (Erasmus, 2001 : 13).

"C'est une chose de situer un sujet ou un peuple comme « Autre » dans un discours dominant; c'en est une autre que de le soumettre à ce «savoir », non seulement au moyen d'une domination et d'une volonté imposées, mais aussi par une contrainte interne et une conformation subjective à la norme. » (Hall, 2007 : 230).

Nous sommes face à une oppression qui n'est pas seulement imposée physiquement (la violence quotidienne émanant des institutions ou du groupe lui-même) ou économiquement (par l'exploitation sur le marché du travail ou la marginalisation sociale) mais également politiquement et symboliquement, ce que I. M. Young (1990) a appelé l'impérialisme culturel (processus d'assignations identitaires) et le disempowerment (obstacles structurels faits à la prise de décision).

S. Jensen insiste sur le rôle de la relative protection sociale accordée sous l'apartheid dans le disempowerment des électeurs 'colorés' aujourd'hui, face au nouveau gouvernement qui tente d'équilibrer la répartition des aides sociales à l'échelle de tous les citoyens et non plus de la minorité "colorée"16. "Diviser pour mieux régner ", la stratégie de la domination blanche laisse en héritage un fort antagonisme entre 'Noirs' et 'Colorés'. Outre l'aggravation du choc économique de la libéralisation postapartheid, la mise sous perfusion des familles 'colorées' a des conséquences identitaires profondes, infantilisant les femmes en leur imposant par exemple des cours sur les travaux et les finances domestiques et stigmatisant les hommes comme délinquants (Badroodien, 1999). Dès l'enfance, les garçons étaient envoyés dans des «reformatories " afin de les protéger d'un univers défini comme violent a priori; à l'âge adulte, le système pénitentiaire prenait le relais. Le taux d'emprisonnement des hommes est bien plus élevé dans les communautés 'colorées' que dans les autres groupes.

Tableau 1 - Taux d'emprisonnement des hommes selon leur groupe racial (en nombre de prisonniers pour 100000 membres de chaque groupe)

\begin{tabular}{|l|l|l|l|l|}
\hline & $\begin{array}{l}1970 \\
\text { (chiffres de la } \\
\text { commission Wilcocks) }\end{array}$ & $\begin{array}{l}1979-1980 \\
\text { (chiffres de de } \\
\text { Pinnock, 1983) }\end{array}$ & $\begin{array}{l}\mathbf{2 0 0 3} \\
\text { (chiffres } \\
\text { Muntingh, 2008) }\end{array}$ & $\begin{array}{l}\mathbf{2 0 0 9} \\
\text { (chiffres de } \\
\text { l'auteure*) }\end{array}$ \\
\hline 'Coloreds' & 791 & 729 & 706 & 633 \\
\hline 'Africans' & 425 & 362 & DM & 332 \\
\hline 'Indians' & 86 & 81 & DM & 52 \\
\hline
\end{tabular}




\begin{tabular}{|l|l|l|l|l|}
\hline 'Whites' & 80 & 57 & DM & 55 \\
\hline
\end{tabular}

* calculés à partir de Stats SA et Department of Correctional Services.

Comme le rappelle Steffen Jensen, le débat n'est pas de savoir si ou pourquoi les 'colorés' sont en effet plus criminels que les autres.

"The way in which state agencies approached a particular group of people influenced the strategies of coping that were open to the particular group. (...) Apartheid practices penetrated the everyday life of coloureds in (...) structural ways. (...) the violence against coloureds was located in social welfare offices and prisons. This kind of violence is, as we know from Foucault (1979), highly political in that it accomplishes the depoliticization of oppression by turning it into wellmeaning intervention, aimed at making the population healthy. » (Jensen, $2008: 36$ \& 39)

21 S. Jensen met en lumière le double jeu entre imposition et intériorisation de l'infériorité et la confusion entre pauvreté et racisme. L'idée d'une dépolitisation inscrite au cœur de la vie quotidienne est un élément crucial pour interroger le positionnement des 'Colorés' dans l'échiquier politique actuel. «Les Coloureds sont-ils voués à demeurer dans les limbes de la politique sud-africaine et les nouveaux dirigeants ont-ils la volonté de s'attaquer délibérément à une politique de démantèlement du racisme (deracialisation)?», demandait Don Mattera en 1994, « porte-parole respecté de la communauté » cité par D.-C. Martin (1998 : 97). Après trois élections présidentielles, la question reste posée. Si l'on s'en tient au constat que la communauté 'colorée' s'exprime politiquement via la DA (affirmation qu'il faudrait bien sûr nuancer), force est de constater que le contenu du discours partisan a peu à voir avec une affirmation identitaire. Bien que la DA soit majoritaire à l'échelle de la métropole et de la province, la trame de son positionnement politique reste le face à face avec l'ANC. Dans son programme, les prescriptions de la bonne gouvernance font valoir un pragmatisme gestionnaire. Au Cap, le modèle de citoyenneté proposé est plus celui d'un contribuable méritant que l'on consulte à l'occasion que celui d'un militant qui participe à la deracialisation en articulant des revendications idéologiques ${ }^{17}$.

L'identité 'colorée' est donc prise dans un positionnement à la fois spatialisé (mythe des forced removals / imagerie du township) et racialisé (infériorité de la miscegenation / supériorité sur les 'Africains'). La notion empruntée à Stuart Hall permet le dialogue entre les divers travaux sur les mécanismes identitaires au Cap et met à jour la mobilisation (l'immobilisation?) potentielle du savoir scientifique. La dernière partie de cet article propose d'écouter comment Eugene renoue les fils de sa vie en manipulant les différents récits exposés précédemment. Après avoir décomposé les discours produits sur la communauté, il s'agit de s'attarder sur la mise en mots de leur quotidien par les habitants eux-mêmes afin de mesurer la stabilité du positionnement racial et spatial.

\section{« Depending on the area you stay in », essai vidéo}

Ce document vidéo est un premier montage d'une durée de 7 minutes réalisé à partir d'une interview de plusieurs heures avec Eugene, tournée entre septembre et novembre 2009. Je connais Eugene depuis 2007 et nous avons passé de longues heures avant d'entamer le tournage de ce qui devrait devenir un film documentaire d'une 
trentaine de minutes abordant la ville de Cape Town à travers l'itinéraire d'un de ses habitants.

Ce média ne peut être affiché ici. Veuillez vous reporter à l'édition en ligne http:// journals.openedition.org/echogeo/11939

Le premier degré de lecture de cette proposition filmique est l'accès aux mots exacts employés par Eugene, au ton et à l'intensité de son discours. Plutôt que de développer un commentaire écrit sur le rôle de la religion dans le positionnement identitaire au Cap, j'ai choisi de mettre en avant la rhétorique évangéliste adoptée par Eugene pour résumer les grandes étapes de sa vie. Même lorsqu'il ne parle pas directement de son lien à la religion, sa foi transparait dans le vocabulaire utilisé. Des expressions comme " être dans le monde » ou «être hors du monde », " guider ses enfants", "le contrôle sur sa vie » et bien sûr, l'opposition entre «le bien et le mal " reflètent la prise en charge de son histoire de vie par les schèmes d'explication des «Églises du Christ » en plein essor. L'écriture sous forme de film ouvre également sur un deuxième type de lecture en ce qu'elle repositionne le chercheur dans la production du discours. Monter un film permet de superposer le récit des images au discours sonore. Le dispositif présenté ici suggère par exemple la gentrification actuelle de BoKaap tout en évoquant l'enfance révolue d'un de ses habitants expulsés au début des années 1980.

\section{Conclusion : la voix du chercheur, du portrait singulier au discours collectif}

Le portrait de Eugene retrace les ambiguités d'un itinéraire archétypal : naissance et petite enfance en plein centre-ville (Bo Kaap), puis, au tournant des années 1980, déménagement forcé sur les Cape Flats, intégration dans le township (Heideveld) via une ascension au sein du gang local (les Junky Funky Kids) et finalement sortie du réseau criminel à travers une "renaissance " évangéliste. Mais au delà de la description factuelle, la vidéo permet de donner une voix aux discours évoqués dans l'article. Après avoir suggéré comment lesquels les chercheurs contribuent à la construction des discours identitaires, je voulais redonner la parole à un individu particulier. Ce qui en ressort est surtout la remarquable adéquation entre les fils d'explication proposés par Eugene pour décrire son itinéraire et les grands récits historiques construits scientifiquement.

En effet lorsque Eugene raconte son enfance entre ses parents alcooliques à Bo Kaap, il répète avec précision les stéréotypes raciaux qui ont conduit à l'attitude paternaliste de l'État envers le groupe 'coloré'. Sa façon d'évoquer son passage par un gang de quartier est performative du discours sur la violence endémique des townships 'colorés' et l'absence de contrôle social sur les adolescents dans ces quartiers en dehors au couple gang/prison. Enfin ce que suggère la « rédemption » évangéliste qui fournit à Eugene la matrice de son récit de vie, c'est aussi son immense difficulté à inventer son propre discours, à être l'auteur de sa propre histoire et non pas seulement le spectateur d'une vie décidée par d'autres. Le tableau de sa vie de famille jonglant entre le gangsta rap et les principes religieux charismatiques révèle finalement l'équivocité irrésolue qui pourrait définir la notion de "minorité", "le ghetto" dont parle Eugene, "l'underground" comme diraient les rappeurs. Car malgré les drames d'une histoire sans pareille, la 
communauté 'colorée' du Cap, entre opprobre et quête de dignité, n'est peut-être pas si différente de bien des groupes sociaux dominés et marginalisés dans toutes les villes du monde ${ }^{18}$.

\section{BIBLIOGRAPHIE}

Adhikari M., 2005. Not White Enough, Not Black Enough. Racial Identity in the South African Coloured Community. Ohio University Press / Double Storey Books, 252 p.

Badroodien A., 1999. A History of the Ottery School of Industries in Cape Town: Issues of Race, Welfare and Social Order in the Period 1937 to 1968. Thèse de Sciences de l'éducation, University of the Western Cape.

Buire C., 2007. Townships du Cap: ce qui fait ville. Justice spatiale et citadinité en Afrique du Sud. Mémoire de Master 2 sous la direction de P. Gervais-Lambony, Université de Paris X - Nanterre, $158 \mathrm{p}$.

Desai A., 2004. The Cape of Good Dope? A post-apartheid story of gangs and vigilantes. étude de cas publiée en ligne par le Centre for Civil Society et le département de Development Studies de UKZN, 33 p. : http://www.nu.ac.za/ccs/files/Desai\%20Pagad \%20Research \%20Report.pdf Ebrahim N., 1999. Noor's story: My life in District Six. District Six Museum, 88 p.

Erasmus, Z. (ed.), 2001. Coloured by History, Shaped by Place. New Perspectives on Coloured Identities in Cape Town. Cape Town, Kwela Books, 224 p.

Filed, S., Meyer, R., Swanson, F., 2007. Imagining the city, memories and culture in Cape Town. Cape Town, HSRC Press, $240 \mathrm{p}$.

Gervais-Lambony P., 1997. L’Afrique du Sud et les Etats voisins. Paris, A. Colin, 256 p.

Gervais-Lambony P., 2003. Territoires citadins, 4 villes africaines. Paris, Belin, $272 \mathrm{p}$.

Goldin I., 1987. Making Race, The Politics and Economics of Coloured Identity in South Africa.

Hall, S., 2007. Identité culturelle et diaspora, inIdentités et cultures. Politiques des Cultural Studies. Paris, éd. Amsterdam, p. 227-241.

Hammet, D. 2009. Local beats to global rhythms: coloured student identity and negotiations of global cultural imports in Cape Town, South Africa. Social \& Cultural Geography, 10 (4).

Hammet, D., Jayawardane, N., 2009. Performing the Primitive in the Postcolony: Nyoni's Kraal in Cape Town. Urban Forum, 20, p. 215-233

Houssay-Holzschuch, M., 1999-a. Le Cap, ville sud-africaine. Ville blanche, vies noires, Paris, L'Harmattan, $276 \mathrm{p}$.

Houssay-Holzschuch, M., 1999-b. Les Métis dans la nouvelle Afrique du Sud : étude de deux problèmes identitaires. L'Espace géographique (2).

Jensen, S., 2008. Gangs, Politics and Dignity in Cape Town. Wits Univ. / Univ. of Chicago Press, 212 p. 
Madzou, L., Bacqué, M.-H., 2009. J'étais un chef de gang suivi de Voyage dans le monde des bandes. Paris, La Découverte, 252 p.

Martin, D.-C., 2008. An Imaginary Ocean: Carnival in Cape Town and the Black Atlantic, in Sansone, L., Soumouni, E., Barry B., Africa, Brazil and the construction of Trans Atlantic Black Identities, Trenton/Asmara, Africa World Press, p. 63-79.

Murray N., Sheperd N., Hall M. Desire lines: space, memory and identity in the post-apartheid city. Routledge, $315 \mathrm{p}$.

Muntingh L., 2008. Youth Crime and violence - some perspectives from the prison reform sector. HSRC, table ronde du 13 mai 2008. Papier disponible en ligne : www.hsrc.ac.za/Document-2791.phtml

Pinnock D., 1984. The brotherhoods: street gangs and state control in South Africa. Cape Town, David Phillip Pub., 116 p.

Pinnock D., 1997. Gangs as a rite of passage, inGangs rituals and rites of passage, African Sun Press, UCT, p. 28-54

Piombo J., Nijzink L. (dir.), 2005. Electoral Politics in South Africa. Assessing the first democratic decade. Cape Town, HSRC Press, $297 \mathrm{p}$.

Posel D., 2001. What's in a name? Racial categorizations under apartheid and their afterlife. Transformation, $\mathrm{n}^{\circ} 47$, p. 50-74

Salo E. 2003. Negotiating gender and personhood in the new South Africa. Adolescent women and gangsters in Manenberg township on the Cape Flats. European Journal of Cultural Studies 6 (3)

Soudien C., 2001. District Six and its uses in the discussion about non-racialism, in Erasmus (eds.),Coloured by History, Shaped by Place. New Perspectives on Coloured Identities in Cape Town. Cape Town, Kwela Books, p. 114-130.

Standing A., 2003. The social contradictions of organised crime on the Cape Flats. Institute for Security Studies, paper $74,12 \mathrm{p}$.

Stone G. L., 1995. The lexicon and socio-linguistic codes of the working class Afrikaans-speaking Cape Peninsula coloured community, in Meshtries R. (ed.), Language and Social History in South African Linguistics, Cape Town, David Philip, p. 227-290.

Steinberg J., 2004. The Number. One man's search for identity in the Cape underworld and prison gangs. Johannesburg / Cape Town, Jonathan Ball Publishers, 440 p.

Western J., 1981. Outcast Cape Town. Berkeley, University of California Press, 372 p.

Witz L., 2007. Museums on Cape Town's Township Tours, inMurray N., Sheperd N., Hall M., Desire lines: space, memory and identity in the post-apartheid city. p. 259-275.

Worden N., Van Heyningen E., Bicford-Smith V., 1998. Cape Town, the making of a city: an illustrated social history. Cape Town, David Philip Pub., 283 p.

Worden N.. Van Heyningen E., Bicford-Smith V., 1999. Cape Town in the twentieth century: an illustrated social history, Cape Town, David Philip Pub., 255 p.

\section{NOTES}

1. Avec $17 \%$ des voix en 2009 contre $66 \%$ pour l'ANC, la DA constitue le premier parti d'opposition à l'échelle nationale. Elle se définit comme un parti social-libéral et revendique des racines dans les mouvements libéraux de lutte contre l'apartheid. Elle a capté la majorité du vote 
blanc, dans un pays où les choix électoraux semblent respecter une répartition plus raciale qu'idéologique (Piombo \& Nijzink, 2005).

2. Au recensement de 2001 , les statistiques officielles répartissaient les 2,9 millions d'habitants de la métropole suivant les proportions suivantes : Coloured $48 \%$; Black African $32 \%$; White $19 \%$; Indian or Asian $1 \%$. Ces désignations reposent sur la déclaration des personnes recensées et non plus sur une catégorisation administrative comme c'était le cas sous l'apartheid. Voir Posel 2001. Dans la suite du texte, des apostrophes signalent l'utilisation de ces termes afin d'insister sur leur construction historique et de se distancier du racisme qui les sous-tend.

3. "A Coloured person is a person who is not a white person nor a native.", extrait du Population Registration Act no. 30 of 1950, section 1, annulé en 1991 (cité par Posel, 2001 : 56).

4. "The challenge for all South Africans is to begin to recognize racist sentiments and practices as part of our everyday reality and the shaping of all our selves. (...) A progressive, transformative politics cannot be based on a denial of the past. The realization that no one South African can claim a moral high ground, that all of us have been profoundly wounded and shaped by the past, is more likely to provide the ground for creating new identities. » (Erasmus, 2001:26). Remarque pour cette citation et pour la suite du texte : toutes les traductions sont proposées par l'auteure à titre indicatif.

5. Dans ce contexte, le terme 'noir' désigne tous les «non blancs ». Je l'utiliserai dans ce sens pour la suite du texte. Cette acception s'est généralisée dans les années 1970 à partir des cercles anti-racistes militant pour un front commun de lutte contre l'apartheid par-delà les divisions raciales intériorisées qui provoquaient la division, en particulier entre Coloureds et Africans. Pour une discussion sur l'adoption du terme 'black' dans la communauté colorée, voir Adhikari 2005.

6. "A white person is one who in appearance is, or is generally accepted as, a white person. (...) A native is a person who is in fact or is generally accepted as a member of any aboriginal race or tribe of Africa. ", extrait du Population Registration Act no. 30 of 1950, section 1, annulé en 1991 (cité par Posel, 2001 : 56).

7. Martin (1998) donne les chiffres suivants : 26,4\% seraient originaires d'Afrique (acheminés via les ports mozambicains ou angolais), 25,1\% de Madagascar (et des îles environnantes), 25,9\% d'Inde (y compris de Ceylan) et 22,7\% de la région des actuelles Malaisie et Indonésie.

8. «By the 1790s, free blacks had to carry passes, in the same way as slaves. It seems that the higherranking echelons of Cape Town society were seeking to exclude the pretentions of those beneath them - and many of them happened out to be of pure European ancestry. The emerging division within the town was between racially more (but by no means completely) exclusive wealthy elite and a poorer and racially more complex population. » (Worden et al., $1998: 70$ )

9. Ces quartiers sont Ndabeni de 1901 à 1936 puis Langa à partir des années 1930.

10. La notion de «fordisme racial » a été lancée par S. Gelb pour décrire la crise économique des années 1980. Gelb décrit comment l'interventionnisme de l'État sud-africain d'après-guerre a permis l'ascension du prolétariat blanc tout en maintenant les travailleurs noirs dans la précarité, les postes qualifiés étant réservés aux ‘Blancs’ (Gelb, 1987).

11. Voir Erasmus, 2001, p. 14 : « This book (...) argues that coloured identities are not based on 'race mixture' but on cultural creativity, creolized formations shaped by South Africa's history of colonialism, slavery, segregation and apartheid. This conceptualization undermines the common sense view that conceives colouredness as something produced by the mixture of other 'purer' cultures. "

12. La présence africaine est définie comme "le lieu du refoulé ", "l'Afrique" telle que nous l'avons re-racontée à travers la politique, la mémoire et le désir ». La présence européenne est « l'exemple de ce qui ne cesse pas de parler, de ce qui n'a de cesse de nous parler ». La présence américaine enfin est celle du "Nouveau Monde », elle "n'est pas tant une présence de pouvoir que de sol, de lieu, de territoire. » (Hall, $2007: 236$-238).

13. " And if the emblematic figure of African modernity in Johannesburg is the migrant worker, a figure that moves from the margins to the underground of the mines then in the case of Cape Town, with its 
history of (limited) racial cohabitation and subsequent segregation through apartheid, the emblematic urban figure is the victim of forced removal. »(Murray, Shepherd, Hall. 2007 :14)

14. Il faut rappeler que les déménagements forcés ont été imposés à tous les non-Blancs et marquent donc l'histoire citadine des résidents 'Africains' autant que des 'Colorés'. Ce qui en fait un récit si central pour le groupe 'coloré' est qu'il n'est que faiblement complété par d'autres expériences comme celles du travail migrant ou des discutables origines "ethniques" imposées aux 'Africains' avec la mise en place des bantoustans.

15. "Popular cultural myths (...) provide people with the vocabulary and beliefs to understand and cope with a myriad of challenges within the city. " (Field, Meyer, Swanson, $2007: 11$ ).

16. En 1990, les 'Colorés' qui représentaient $9 \%$ de la population recevaient $50 \%$ des pensions gouvernementales (state maintenance), soit 50 allocations pour 1000 enfants contre seulement 2 pour les 'Africains' et 15 pour les 'Blancs'. (Jensen, $2008: 38-39$ )

17. La discussion sur la citoyenneté ici ébauchée fait référence à mon travail de thèse en cours soutenu par le programme CORUS, «La voix des pauvres dans la gouvernance urbaine en Afrique du Sud» dirigé par C. Bénit-Gbaffou (Gecko, Université de Nanterre) et A. Mabin (Cubes, Witswatersrand University) dont je tiens à remercier ici toute l'équipe.

18. Une première piste comparatiste émerge de la lecture parallèle du livre de Jonny Steinberg sur l'univers des gangs au Cap et le témoignage de Lamence Madzou commenté par Marie-Hélène Bacqué sur les bandes de la région parisienne dans les années 1980 - 1990 (Steinberg, 2004 ; Madzou \& Bacqué, 2009).

\section{RÉSUMÉS}

Le Cap est la "Ville-Mère" de la "Nation Arc-en-Ciel". Aujourd'hui la majorité des habitants du Cap est considérée 'Coloured'. L'article remonte le fil de la construction de cette catégorie qui regroupe ceux qui ne sont "ni assez Blancs, ni assez Noirs» (Adhikari, 2005). La notion de positionnement identitaire empruntée à Stuart Hall permet d'articuler deux grands récits : le traumatisme des déménagements forcés et le double complexe de la hiérarchie raciale. Un court portrait vidéo montre finalement comment un individu mobilise ces discours scientifiques avec pragmatisme. La parole d'Eugene, baignée de rhétorique évangéliste, invite à interroger le statut de nos propres discours scientifiques et leur rôle dans l'édification des stéréotypes raciaux.

Cape Town is the "Mother-City" of the "Rainbow Nation". Today the majority of its population is considered 'Coloured'. The article disentangles the course of the construction of such a category which gather those who are "not White enough, not Black enough" (Adhikari, 2005). Stuart Hall's notion of positioning helps to articulate two meta-narratives: the trauma caused by forced removals and the double-edge complex encapsulated by racial hierarchy. A short video portrait eventually shows how an individual pragmatically makes use of those scientific discourses. Eugene's words, shaped in evangelist rhetoric are an invitation to question the status of our own scientific discourses and their role in building racial stereotypes. 
INDEX

Keywords : Cape Town, identity, narratives, racism, video

Mots-clés : discours, identité, Le Cap, racisme, vidéo

\section{AUTEUR}

\section{CHLOÉ BUIRE}

Chloé Buire est doctorante en géographie, Laboratoire GECKO, Université Paris Ouest - Nanterre. Ses dernières publications :

- Buire C., 2008. 'On the ground', de la déconstruction de l'idée de terrain à la construction de savoirs géographiques. Manuscrit auteur publié en ligne parmi les textes du colloque «A travers l'espace de la méthode : les dimensions du terrain en géographie », Arras 18-20 juin. http:// halshs.archives-ouvertes.fr/halshs-00421957/fr/

- Buire C., 2009. Inventer la citoyenneté après l'apartheid: les tactiques des habitants pauvres face à la 'bonne gouvernance' - Cape Town, Afrique du Sud. Territoires (497), p. 38-41.

- Essahel H., Buire C., Guéye A., Fendris., Mouaziz N., Dridi N., Mouloudi H., 2009. La négociation comme mode de fabrication des territoires urbains. Réflexions à partir de quelques cas au Maroc, en Algérie, en Tunisie, au Sénégal et en Afrique du Sud. Actes de la Rencontre Jeunes Chercheurs, programme FSP. Oran 4-7 avril 2009. http://citeres.univ-tours.fr//p_emam/contrats/fsp/ fichier6.pdf .

chloebuire@gmail.com 